\title{
Application of sevoflurane inhalation combined with epidural anesthesia in patients with colorectal cancer and its effect on postoperative perceptual function
}

\author{
XIAOMIN ZHANG ${ }^{1}$ and $\mathrm{HAO}_{\mathrm{JIANG}}^{2}$ \\ ${ }^{1}$ Department of Anesthesia, The Second Cancer Hospital of Heilongjiang Province, Harbin, Heilongjiang 150086; \\ ${ }^{2}$ Department of Anesthesia, Harbin Medical University Cancer Hospital, Harbin, Heilongjiang 150081, P.R. China
}

Received October 12, 2018; Accepted February 19, 2019

DOI: $10.3892 / \mathrm{ol} .2019 .10074$

\begin{abstract}
Effect of sevoflurane inhalation combined with epidural anesthesia on postoperative perceptual function in patients with colorectal cancer (CRC) was investigated. The clinical data of 78 patients undergoing laparoscopic CRC radical surgery in The Second Cancer Hospital of Heilongjiang Province from June 2016 to June 2017 were retrospectively analyzed and divided into 2 groups: Sevoflurane inhalation combined with epidural anesthesia (observation group, $n=40$ ); propofol intravenous general anesthesia (control group, $n=38$ ). The mean arterial pressure and heart rate before anesthesia (T0), immediate intubation (T1), 30 min after surgery started (T2), end of surgery (T3), 10 min after extubation (T4) were compared between the two groups. The recovery conditions were also compared between the two groups. The cognitive functions were evaluated by mini-mental state scale (MMSE). The mean arterial pressure and heart rate in the observation group were significantly lower than those in the control group at $\mathrm{T} 2, \mathrm{~T} 3$ and $\mathrm{T} 4(\mathrm{P}<0.05)$. The recovery time, extubation time, anal exhaust time, eating time, urinary catheter removal time and hospital stay in the observation group were significantly lower than those in the control group $(\mathrm{P}<0.05)$. There was a significant difference in cognitive function between the two groups at different time points $(\mathrm{P}<0.001)$. Postoperative cognitive function showed a trend of decreasing first and then increasing; the scores of cognitive function in both groups 1 day after surgery were at trough level, and recovered gradually from 3 days after surgery. The mean arterial pressure and heart rate during the perioperative period are more stable in the elderly patients with sevoflurane inhalation combined
\end{abstract}

Correspondence to: Dr Hao Jiang, Department of Anesthesia, Harbin Medical University Cancer Hospital, 150 Haping Road, Harbin, Heilongjiang 150081, P.R. China

E-mail: hqz4qw@163.com

Key words: sevoflurane, epidural, anesthesia, colorectal cancer, cognitive functions with epidural anesthesia; the recovery time is shorter and more rapid, and the recovery time of postoperative cognitive function is also faster. Therefore, it provides a reference for patients undergoing CRC radical surgery to select high-quality and appropriate anesthetic protocols.

\section{Introduction}

Colorectal cancer (CRC) is the third most common malignant tumor in the world and the fourth leading cause of cancer death, with approximately 1.4 million new cases and nearly 700,000 deaths in 2012 (1). Resection is one of the most important methods for the treatment of early CRC in clinical practice. Laparoscopic CRC radical surgery is widely used in clinic because of its clear vision, less trauma, less immune system interference, rapid postoperative recovery, and low overall operating costs (2). General anesthesia is required during laparoscopic radical surgery to avoid the effect of artificial pneumoperitoneum on respiratory and circulatory function of patients (3), and postoperative cognitive impairment may be caused after surgery and anesthesia in the elderly, which can affect the postoperative recovery and quality of life (4). Therefore, in order to reduce the incidence of postoperative cognitive impairment, appropriate anesthesia methods should be selected.

There are two main techniques for anesthesia: i) General anesthesia, where gas or intravenous drugs achieve central nervous system depression and ii) local anesthesia, where the drug is directly administered to the spinal cord or nerve to locally block the input of afferent and efferent nerve (5). Propofol for total intravenous injection and sevoflurane for inhalation anesthesia are mainly used in general anesthesia because their pharmacological properties facilitate rapid recovery after anesthesia (6-8). Propofol is superior in recovering features and reducing postoperative adverse reactions (9). Sevoflurane is volatile, nonflammable and aromatic, non-irritating to the respiratory tract, and has low blood gas partition coefficient, which is conducive to the regulation of anesthesia depth, smooth induction and rapid recovery (10). However, sevoflurane is superior to propofol in anesthetic applications requiring rapid induction and recovery of cognitive function $(11,12)$. 
Epidural anesthesia and analgesia are commonly used to manage postoperative pain after abdominal surgery. Afferent block induced by epidural anesthesia can reduce neuroendocrine stress during and after surgery $(13,14)$. Epidural anesthesia can reduce neuroendocrine stress and prevent immune suppression caused by surgery and general anesthesia, thus protecting patients from complications after infection or postoperative development of tumor metastasis $(15,16)$.

This study analyzed the application of sevoflurane inhalation combined with epidural anesthesia in CRC radical surgery and its effect on cognitive function of elderly patients, and to provide references for the selection of clinical anesthetic methods in elderly patients.

\section{Patients and methods}

Patient information. The clinical data of 78 patients undergoing laparoscopic CRC radical surgery in The Second Cancer Hospital of Heilongjiang Province (Harbin, China) from June 2016 to June 2017 were retrospectively analyzed, including 44 males and 34 females, and with an age range of 60-75 years. Among them, 40 patients received sevoflurane inhalation combined with epidural anesthesia as observation group; 38 patients received propofol general anesthesia as control group. There was no significant difference between the two groups in general data $(\mathrm{P}>0.05)$. All patients were diagnosed with colon cancer or rectal cancer by Pathology Department of The Second Cancer Hospital of Heilongjiang Province. Patients with other malignant tumors, heart, liver and kidney dysfunction, coagulation dysfunction, mental illness and incomplete clinical data were excluded.

The study was approved by the Ethics Committee of The Second Cancer Hospital of Heilongjiang Province. Patients who participated in this research, signed an informed consent and had complete clinical data. Patient information is provided in Table I.

Anesthetic methods. All patients were prohibited from eating $8 \mathrm{~h}$ before surgery and using drugs before surgery. $0.1 \mathrm{~g}$ of luminal (Tianjin Kingyork Pharmaceutical Co., Ltd., Tianjin, China, SFDA approval no. H12020381), and $0.3 \mathrm{mg}$ of scopolamine (Shanghai Harvest Pharmaceutical Co., Ltd., Shanghai, China, SFDA approval no. H31021519) were intramuscularly injected 30 min before surgery. Invasive arterial pressure, central venous pressure, electrocardiogram, blood pressure, heart rate, pulse and oxygen protection monitoring were established, and intravenous channel was established to infuse $500 \mathrm{ml}$ of succinyl gelatin (Shenyang Beilang Pharmaceutical Co., Ltd., Shenyang, China, SFDA approval no. H20040609). Midazolam (0.04 mg/ kg; Jiangsu Enhua Pharmaceutical Group Co., Ltd., Jiangsu, China, SFDA approval no. H20031037), 2-4 $\mu \mathrm{g} / \mathrm{kg}$ fentanyl (Yichang Humanwell Pharmaceutical Co., Ltd., Yichang, China, SFDA approval no. H42022076), $0.1 \mathrm{mg} / \mathrm{kg}$ vecuronium bromide (Zhejiang Xianju Pharmaceutical Co., Ltd., Zhejiang, China, SFDA approval no. H19991172), and $1 \mathrm{mg} / \mathrm{kg}$ propofol (Xi'an Libang Pharmaceutical Co., Ltd., Xi'an, China, SFDA approval no. H20010368) were used for anesthesia induction in both groups. Anesthesia apparatus was connected to control breathing after intubation. In the control group, propofol was pumped 4-6 mg/kg per hour; fentanyl and vecuronium was

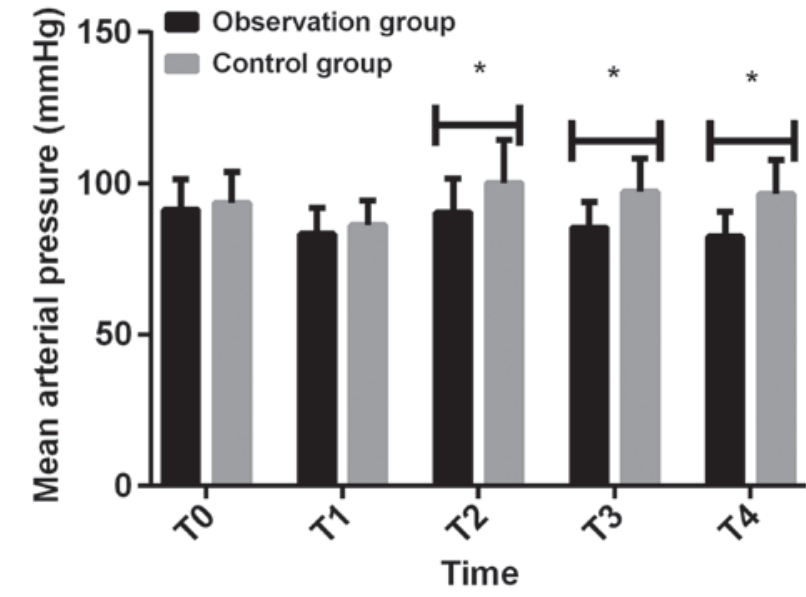

Figure 1. Comparison of mean arterial pressure at different time points between the two groups. The results showed that there was no significant difference in mean arterial pressure between the observation group and the control group at T0 and T1 $(\mathrm{P}>0.05)$; the mean arterial pressure in the observation group was significantly lower than that in the control group at T2, T3 and $\mathrm{T} 4$, and the difference was statistically significant $\left({ }^{*} \mathrm{P}<0.05\right)$.

injected intermittently to maintain anesthesia. In the observation group, epidural puncture was performed before anesthesia induction; $3 \mathrm{ml}$ of the mixture of $1 \%$ lidocaine (Shanghai Chaohui Pharmaceutical Co., Ltd., Shanghai, China, SFDA approval no. H31021071) and $0.25 \%$ ropivacaine (Guangdong Resources Shunfeng Pharmaceutical Co., Ltd., Guangdong, China, SFDA approval no. H20050325) were injected for the first time. After 10 min of observation, additional dose was injected after confirmation of no signs of spinal esthesia. Anesthesia maintenance: In the observation group, an additional dose was injected into the epidural every 45-60 min. Intermittent intravenous injection of vecuronium bromide was conducted and 2-3\% sevoflurane (Fujian Gutian Pharmaceutical Co., Ltd., Fujian, China, SFDA approval no. H35020148) was inhaled to maintain anesthesia, and sevoflurane was discontinued during skin suture.

Observation index. The mean arterial pressure and heart rate before anesthesia (T0), immediate intubation (T1), $30 \mathrm{~min}$ after surgery started (T2), end of surgery (T3), 10 min after extubation (T4) were compared between the two groups.

The recovery conditions including recovery time, extubation time, anal exhaust time, eating time, urinary catheter removal time and hospital stay were compared between the two groups.

The cognitive functions, including orientation, attention, calculation, reading, comprehension, memory, were evaluated by mini-mental state scale (MMSE) at 1 day, 3 days and 5 days after surgery, respectively. The total score was 30 , and the smaller the score, the worse the cognitive function.

Statistical analysis. SPSS 20.0 statistical software (IBM Corp., Armonk, NY, USA) was used for analysis. Chi-square test was used for enumeration data, and t-test was used for measurement data. One-way ANOVA with Dunnett's test was used for multi-group comparison, and repeated measures ANOVA was used for comparisons at different times within the group. $\mathrm{P}<0.05$ was considered to indicate a statistically significant difference. 
Table I. Patient information [n (\%)].

\begin{tabular}{|c|c|c|c|c|}
\hline Factor & $\begin{array}{l}\text { Observation group } \\
\qquad(\mathrm{n}=40)\end{array}$ & $\begin{array}{l}\text { Control group } \\
\qquad(\mathrm{n}=38)\end{array}$ & $\mathrm{t} / \chi^{2}$ value & P-value \\
\hline Age (years) & $67.63 \pm 6.22$ & $66.07 \pm 7.14$ & 1.030 & 0.306 \\
\hline Sex & & & 0.430 & 0.648 \\
\hline Male & $24(60.00)$ & $20(52.63)$ & & \\
\hline Female & $16(40.00)$ & $18(47.37)$ & & \\
\hline $\mathrm{BMI}\left(\mathrm{kg} / \mathrm{m}^{2}\right)$ & $23.46 \pm 1.92$ & $23.83 \pm 2.14$ & 0.805 & 0.424 \\
\hline Surgical duration (min) & $218.82 \pm 45.19$ & $212.53 \pm 49.37$ & 0.587 & 0.559 \\
\hline Intraoperative bleeding volume (ml) & $207.56 \pm 36.24$ & $216.22 \pm 34.53$ & 1.079 & 0.284 \\
\hline Rehydration (ml) & $2587.27 \pm 14.05$ & $2613.54 \pm 166.77$ & 0.993 & 0.324 \\
\hline ASA classification [(n\%)] & & & 0.781 & 0.469 \\
\hline $\mathrm{I}$ & $29(72.50)$ & $24(63.16)$ & & \\
\hline II & $11(27.50)$ & $14(36.84)$ & & \\
\hline Education degree $[(\mathrm{n} \%)]$ & & & 2.000 & 0.204 \\
\hline Primary and below & $32(80.00)$ & $25(65.79)$ & & \\
\hline Above primary & $8(20.00)$ & $13(34.21)$ & & \\
\hline
\end{tabular}

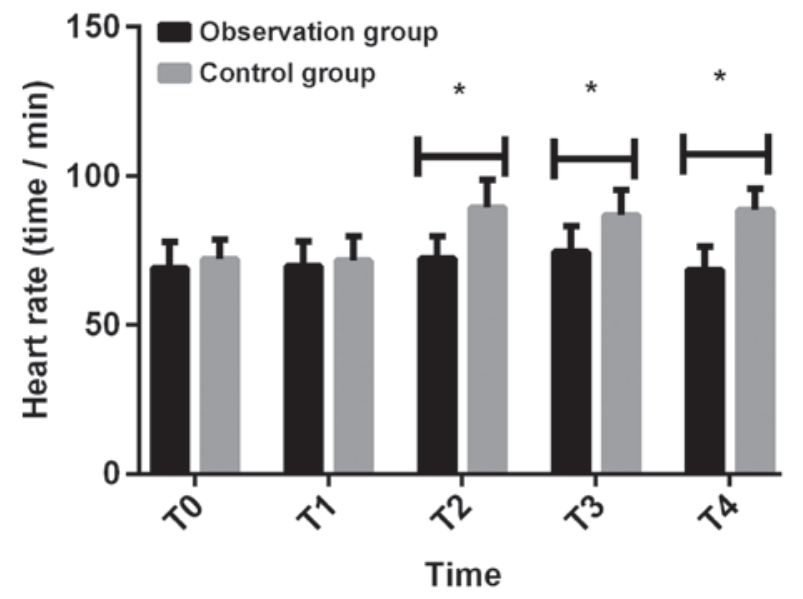

Figure 2. Comparison of heart rate at different time points between the two groups. The results showed that there was no significant difference in heart rate between the observation group and the control group at T0 and T1 $(\mathrm{P}>0.05)$. Heart rate in observation group was significantly lower than that in control group at $\mathrm{T} 2, \mathrm{~T} 3$ and $\mathrm{T} 4$, and the difference was statistically significant $(\mathrm{P}<0.05) .{ }^{*} \mathrm{P}<0.05$.

\section{Results}

Comparison of mean arterial pressure and heart rate at different time points between the two groups. There was no significant difference in mean arterial pressure and heart rate between the observation group and the control group at T0 and $\mathrm{T} 1$ ( $\mathrm{P}>0.05)$, while the mean arterial pressure and heart rate in the observation group were significantly lower than those in the control group at T2, T3 and T4, and the difference was statistically significant $(\mathrm{P}<0.05)$ (Figs. 1 and 2, and Tables II and III.

Comparison of the recovery conditions between the two groups. The recovery time, extubation time, anal exhaust time, eating time, urinary catheter removal time and hospital stay in
Table II. Mean arterial pressure at different time points in the two groups (mmHg).

\begin{tabular}{|c|c|c|c|c|}
\hline Time & $\begin{array}{l}\text { Observation } \\
\text { group } \\
(\mathrm{n}=40)\end{array}$ & $\begin{array}{l}\text { Control } \\
\text { group } \\
(n=38)\end{array}$ & $\mathrm{t}$ value & P-value \\
\hline T0 & $91.26 \pm 10.14$ & $93.56 \pm 10.32$ & 0.993 & 0.324 \\
\hline $\mathrm{T} 1$ & $83.37 \pm 8.53$ & $86.14 \pm 8.25$ & 1.457 & 0.149 \\
\hline $\mathrm{T} 2$ & $90.42 \pm 11.29$ & $100.21 \pm 14.25$ & 3.372 & 0.001 \\
\hline $\mathrm{T} 3$ & $85.38 \pm 8.51$ & $97.26 \pm 10.91$ & 5.377 & $<0.001$ \\
\hline $\mathrm{T} 4$ & $82.43 \pm 8.27$ & $96.57 \pm 11.28$ & 6.336 & $<0.001$ \\
\hline
\end{tabular}

Table III. Heart rate at different time points in the two groups (times/min).

\begin{tabular}{lccrr}
\hline & $\begin{array}{c}\text { Observation } \\
\text { group } \\
\text { Time }\end{array}$ & $\begin{array}{c}\text { Control } \\
\text { group } \\
(\mathrm{n}=38)\end{array}$ & t value & P-value \\
\hline T0 & $69.24 \pm 8.63$ & $72.16 \pm 6.51$ & 1.680 & 0.097 \\
T1 & $69.86 \pm 8.25$ & $71.56 \pm 8.11$ & 0.917 & 0.362 \\
T2 & $72.37 \pm 7.38$ & $89.42 \pm 9.26$ & 9.016 & $<0.001$ \\
T3 & $74.53 \pm 8.62$ & $86.79 \pm 8.51$ & 6.318 & $<0.001$ \\
T4 & $68.62 \pm 7.64$ & $88.58 \pm 7.25$ & 11.820 & $<0.001$ \\
\hline
\end{tabular}

the observation group were significantly lower than those in the control group, and the difference was statistically significant $(\mathrm{P}<0.05)$ (Fig. 3 and Table IV).

Comparison of postoperative cognitive functions between the two groups. There was no significant difference in cognitive 
Table IV. Comparison of the recovery conditions between the two groups.

\begin{tabular}{lccrr}
\hline Time & $\begin{array}{c}\text { Observation group } \\
(\mathrm{n}=40)\end{array}$ & $\begin{array}{c}\text { Control group } \\
(\mathrm{n}=38)\end{array}$ & t value & P-value \\
\hline Recovery time (min) & $7.38 \pm 2.64$ & $14.62 \pm 4.37$ & 8.908 & $<0.001$ \\
Extubation time (min) & $8.13 \pm 2.98$ & $19.57 \pm 5.38$ & 11.690 & $<0.001$ \\
Anal exhaust time (days) & $2.37 \pm 0.87$ & $3.14 \pm 0.97$ & 3.695 & $<0.001$ \\
Eating time (days) & $2.51 \pm 0.95$ & $3.21 \pm 1.02$ & 3.138 & 0.002 \\
Urinary catheter removal time (days) & $1.29 \pm 0.43$ & $1.77 \pm 0.32$ & 5.570 & $<0.001$ \\
Hospital stay (days) & $9.26 \pm 2.45$ & $12.07 \pm 2.14$ & 5.383 & $<0.001$ \\
\hline
\end{tabular}

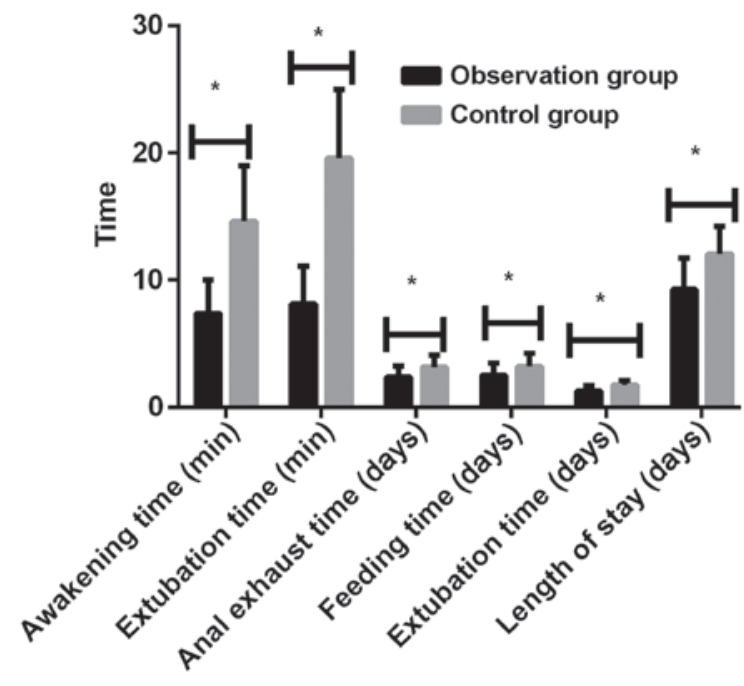

Figure 3. Comparison of the recovery conditions between the two groups The results showed that the recovery time, extubation time, anal exhaust time, eating time, urinary catheter removal time and hospital stay in the observation group were significantly lower than those in the control group, and the difference was statistically significant $\left({ }^{*} \mathrm{P}<0.05\right)$.

functions between the two groups before anesthesia $(\mathrm{P}>0.05)$, but there was a significant difference in cognitive functions between the two groups at different time points $(\mathrm{P}<0.001)$. There was no significant difference in cognitive functions between the two groups before anesthesia and 5 days after anesthesia $(\mathrm{P}>0.05)$; the cognitive functions at 1 and 3 days after surgery was significantly lower than that before anesthesia; 3 days and 5 days after surgery was significantly higher than 1 day after surgery, and 5 days after surgery was significantly higher than 3 days after surgery, and the differences were statistically significant $(\mathrm{P}<0.05)$ (Fig. 4 and Table V).

\section{Discussion}

Life and death are the normal state of life, and the function of the organ is irreversibly degraded with age, and the morbidity and mortality of elderly patients are also affected by this physiological phenomenon (17). With the development of aging society, the number of elderly patients with CRC have increased gradually (18). According to literature, carbon dioxide pneumoperitoneum may threaten the life of patients undergoing laparoscopic surgery $(19,20)$. In particular, the postoperative recovery of elderly patients is often affected

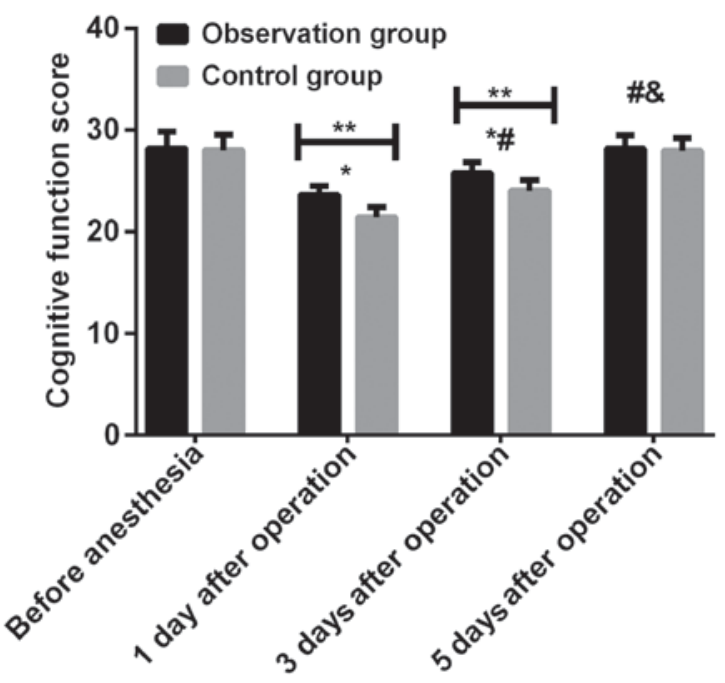

Figure 4. Comparison of postoperative cognitive functions between the two groups. The results showed that there was no significant difference in cognitive functions between the two groups before anesthesia $(\mathrm{P}>0.05)$, but there was a significant difference in cognitive functions between the two groups at different time points $(\mathrm{P}<0.001)$. There was no significant difference in cognitive functions between the two groups before anesthesia and 5 days after anesthesia $(P>0.05)$; the cognitive functions at 1 day and 3 days after surgery was significantly lower than that before anesthesia; 3 days and 5 days after surgery was significantly higher than 1 day after surgery, and 5 days after surgery was significantly higher than 3 days after surgery; the differences were statistically significant $(\mathrm{P}<0.05)$. ${ }^{* *} \mathrm{P}<0.05 ;{ }^{*} \mathrm{P}<0.05$ compared with before anesthesia; ${ }^{\#} \mathrm{P}<0.05$ compared with 1 day after surgery; ${ }^{\&} \mathrm{P}<0.05$ compared with 3 days after surgery.

by anesthesia and other factors (21), and it can also directly affect the cost of treatment, length of stay, and morbidity (22). According to reports in the literature, the proportion of elderly patients who have experienced unconsciousness after major surgery has reached $20 \%(23,24)$. At present, the pathogenesis of postoperative cognitive dysfunction has not been clarified, but a large number of comprehensive studies have shown that it may be related to anesthesia, patient age, type of surgery, education degree and other factors (25-27). In elderly patients, the immune function is reduced due to the gradual degradation of organ function in the body, and the body's ability to metabolize anesthetic drugs is also slower, resulting in a longer recovery time (28). Therefore, in order to make the elderly patients recover as soon as possible after anesthesia, safe, fast metabolized volatile anesthetics should be chosen to avoid the occurrence of postoperative cognitive dysfunction. 
Table V. Comparison of postoperative cognitive functions between the two groups.

\begin{tabular}{lccr}
\hline Time & $\begin{array}{c}\text { Observation group } \\
(\mathrm{n}=40)\end{array}$ & $\begin{array}{c}\text { Control group } \\
(\mathrm{n}=38)\end{array}$ & $\begin{array}{r}\text { P-value } \\
\text { t value }\end{array}$ \\
\hline Before anesthesia & $28.19 \pm 1.64$ & $28.01 \pm 1.52$ & 0.502 \\
1 day after surgery & $23.64 \pm 0.87^{\mathrm{a}}$ & $21.41 \pm 1.05^{\mathrm{a}}$ & 10.230 \\
3 days after surgery & $25.76 \pm 1.07^{\mathrm{a}, \mathrm{b}}$ & $24.01 \pm 1.03^{\mathrm{a}, \mathrm{b}}$ & 7.352 \\
5 days after surgery & $28.15 \pm 1.32^{\mathrm{b}, \mathrm{c}}$ & $27.96 \pm 1.24^{\mathrm{b}, \mathrm{c}}$ & 0.654 \\
F value & 120.300 & 263.000 & $<0.001$ \\
P-value & $<0.001$ & $<0.001$ & 0.515 \\
\hline
\end{tabular}

${ }^{\text {a }}<0.05$ compared with before anesthesia; ${ }^{b} \mathrm{P}<0.05$ compared with 1 day after surgery; ${ }^{\mathrm{c}} \mathrm{P}<0.05$ compared with 3 days after surgery.

The results showed that there was no significant difference in mean arterial pressure and heart rate between the observation and the control group at $\mathrm{T} 0$ and $\mathrm{T} 1(\mathrm{P}>0.05)$. Although the mean arterial pressure and heart rate in the observation group were significantly lower than those in the control group at T2, T3 and $\mathrm{T} 4$, the hemodynamic parameters in the two groups were stable and normal. The recovery time, extubation time, anal exhaust time, eating time, urinary catheter removal time and hospital stay in the observation group were significantly lower than those in the control group, and the difference was statistically significant $(\mathrm{P}<0.05)$. The findings of Zhang et al (29) were basically consistent with ours, which indicated that sevoflurane inhalation general anesthesia combined with epidural block resulted in more stable hemodynamics and shorter recovery time. At the end of surgery, due to reducing the amount of general anesthesia and relying on epidural anesthesia to complete the final abdominal closure, suture skin, the postoperative pain was alleviated; extubation time was brought forward, and the recovery time was accelerated. Study of Akarsu Ayazoğlu et al (30) also showed that general anesthesia combined with epidural block could make hemodynamics more stable and recovery time shorter. In the study of Nishikawa et al (31), it was once again demonstrated that sevoflurane might be superior to propofol for general anesthesia and epidural analgesia in elderly patients undergoing long-term laparoscopic surgery, with less impact on patients' mental function. Murata et al (32) found that long-term high dose intravenous infusion of propofol changed pharmacokinetics and enhanced analgesic effect, thus prolonging the time of recovery and tracheal intubation extubation.

MMSE can effectively evaluate the cognitive function of the patient's brain, has high effectiveness and credibility, and the operation is relatively simple (33). Therefore, MMSE was used to evaluate the postoperative cognitive functions of the two groups in this study, and the results showed that, there was no significant difference in cognitive function between the two groups before anesthesia $(\mathrm{P}>0.05)$, but there was a significant difference in cognitive function between the two groups at different time points $(\mathrm{P}<0.001)$. Postoperative cognitive function showed a trend of decreasing first and then increasing; the scores of cognitive function in both groups 1 day after surgery were at trough level, and recovered gradually from 3 days after surgery. It speculated that sevoflurane inhalation anesthesia combined with epidural block can accelerate the metabolism of anesthetics and reduce the damage of residual anesthetics to the central nervous system. The study of $\mathrm{Fu}$ and $\mathrm{Li}$ (34), showed that general anesthesia combined with epidural anesthesia had a faster recovery of postoperative cognitive function than that of general anesthesia alone in dystocia women, which is basically consistent with our research results.

However, there are some shortcomings in this study, such as the small number of cases, so the results and conclusions of this study still need to be verified by more research and larger clinical data. We also hope that this study will promote the participation of more scholars in the study of anesthesia in patients undergoing CRC radical surgery.

In conclusion, the mean arterial pressure and heart rate during the perioperative period are more stable in the elderly patients with sevoflurane inhalation combined with epidural anesthesia; the recovery time is shorter and more rapid, and the recovery time of postoperative cognitive function is also faster. Therefore, it provides a reference for patients undergoing $\mathrm{CRC}$ radical surgery to select high-quality and appropriate anesthetic protocols.

\section{Acknowledgements}

Not applicable.

\section{Funding}

No funding was received.

\section{Availability of data and materials}

The datasets used and/or analyzed during the present study are available from the corresponding author on reasonable request.

\section{Authors' contributions}

XZ helped with anesthetic methods and wrote the manuscript. $\mathrm{XZ}$ and $\mathrm{HJ}$ collected and interpreted the observation index. Both authors read and approved the final manuscript.

\section{Ethics approval and consent to participate}

The study was approved by the Ethics Committee of The Second Cancer Hospital of Heilongjiang Province (Harbin, 
China). Patients who participated in this research had complete clinical data. Signed written informed consents were obtained from the patients and/or guardians.

\section{Patient consent for publication}

Not applicable.

\section{Competing interests}

The authors declare that they have no competing interests.

\section{References}

1. Arnold M, Sierra MS, Laversanne M, Soerjomataram I, Jemal A and Bray F: Global patterns and trends in colorectal cancer incidence and mortality. Gut 66: 683-691, 2017.

2. Huang C, Shen JC, Zhang J, Jiang T, Wu WD, Cao J, Huang KJ and Qiu ZJ: Clinical comparison of laparoscopy vs open surgery in a radical operation for rectal cancer: A retrospective case-control study. World J Gastroenterol 21: 13532-13541, 2015.

3. Ihnát P, Martínek L, Mitták M, Vávra P, Ihnát Rudinská L and Zonča P: Quality of life after laparoscopic and open resection of colorectal cancer. Dig Surg 31: 161-168, 2014.

4. Kotekar N, Kuruvilla CS and Murthy V: Post-operative cognitive dysfunction in the elderly: A prospective clinical study. Indian J Anaesth 58: 263-268, 2014.

5. Rodgers A, Walker N, Schug S, McKee A, Kehlet H, van Zundert A, Sage D, Futter M, Saville G, Clark T, et al Reduction of postoperative mortality and morbidity with epidural or spinal anaesthesia: Results from overview of randomised trials. BMJ 321: 1493, 2000.

6. Hofer CK, Zollinger A, Büchi S, Klaghofer R, Serafino D, Bühlmann S, Buddeberg C, Pasch T and Spahn DR: Patient well-being after general anaesthesia: A prospective, randomized, controlled multi-centre trial comparing intravenous and inhalation anaesthesia. Br J Anaesth 91: 631-637, 2003.

7. Joo HS and Perks WJ: Sevoflurane versus propofol for anesthetic induction: A meta-analysis. Anesth Analg 91: 213-219, 2000.

8. Godet G, Watremez C, El Kettani C, Soriano C and Coriat P: A comparison of sevoflurane, target-controlled infusion propofol, and propofol/isoflurane anesthesia in patients undergoing carotid surgery: A quality of anesthesia and recovery profile. Anesth Analg 93: 560-565, 2001.

9. Jellish WS, Lien CA, Fontenot HJ and Hall R: The comparative effects of sevoflurane versus propofol in the induction and maintenance of anesthesia in adult patients. Anesth Analg 82: 479-485, 1996.

10. Lokhat D, Golandaj A and Ramjugernath D: Scale-up of sevoflurane synthesis: Selection of chemical route and influence of reagent characteristics. Int J Appl Chem 12: 455-461, 2016.

11. Schoen J, Husemann L, Tiemeyer C, Lueloh A, Sedemund-Adib B, Berger KU, Hueppe M and Heringlake M: Cognitive function after sevoflurane- vs propofol-based anaesthesia for on-pump cardiac surgery: A randomized controlled trial. Br J Anaesth 106: 840-850, 2011.

12. Goswami U, Babbar S and Tiwari S: Comparative evaluation of the effects of propofol and sevoflurane on cognitive function and memory in patients undergoing laparoscopic cholecystectomy: A randomised prospective study. Indian J Anaesth 59: 150-155, 2015.

13. Gupta A, Björnsson A, Fredriksson M, Hallböök O and Eintrei C: Reduction in mortality after epidural anaesthesia and analgesia in patients undergoing rectal but not colonic cancer surgery: A retrospective analysis of data from 655 patients in central Sweden. Br J Anaesth 107: 164-170, 2011

14. Kehlet H: Manipulation of the metabolic response in clinical practice. World J Surg 24: 690-695, 2000.

15. Snyder GL and Greenberg S: Effect of anaesthetic technique and other perioperative factors on cancer recurrence. Br J Anaesth 105: 106-115, 2010.

16. Ahlers O, Nachtigall I, Lenze J, Goldmann A, Schulte E, Höhne C, Fritz G and Keh D: Intraoperative thoracic epidural anaesthesia attenuates stress-induced immunosuppression in patients undergoing major abdominal surgery. Br J Anaesth 101: 781-787, 2008.
17. King MS: Preoperative evaluation of the elderly. J Am Board Fam Pract 4: 251-258, 1991.

18. Miller KD, Siegel RL, Lin CC, Mariotto AB, Kramer JL, Rowland JH, Stein KD, Alteri R and Jemal A: Cancer treatment and survivorship statistics, 2016. CA Cancer J Clin 66: 271-289, 2016.

19. Bhattacharjee DP, Saha S, Paul S, Roychowdhary S, Mondal S and Paul S: A comparative study of esmolol and dexmedetomidine on hemodynamic responses to carbon dioxide pneumoperitoneum during laparoscopic surgery. Anesth Essays Res 10: 580-584, 2016.

20. Mutetwa EN, Shumbairerwa S, Crawford A, Madzimbamuto FD, Chimoga T and Marange-Chikuni D: Metabolic effects of Carbon Dioxide $\left(\mathrm{CO}_{2}\right)$ insufflation during laparoscopic surgery: Changes in $\mathrm{pH}$, arterial partial Pressure of Carbon Dioxide $\left(\mathrm{PaCO}_{2}\right)$ and End Tidal Carbon Dioxide (EtCO $\left.{ }_{2}\right)$. Cent Afr J Med 61: 61-65, 2015.

21. Weingarten TN, Bergan TS, Narr BJ, Schroeder DR and Sprung J: Effects of changes in intraoperative management on recovery from anesthesia: A review of practice improvement initiative. BMC Anesthesiol 15: 54, 2015.

22. Thiele RH, Rea KM, Turrentine FE, Friel CM, Hassinger TE, McMurry TL, Goudreau BJ, Umapathi BA, Kron IL, Sawyer RG, et al: Standardization of care: Impact of an enhanced recovery protocol on length of stay, complications, and direct costs after colorectal surgery. J Am Coll Surg 220: 430-443, 2015.

23. Rudolph JL, Marcantonio ER, Culley DJ, Silverstein JH, Rasmussen LS, Crosby GJ and Inouye SK: Delirium is associated with early postoperative cognitive dysfunction. Anaesthesia 63: 941-947, 2008.

24. Saczynski JS, Marcantonio ER, Quach L, Fong TG, Gross A, Inouye SK and Jones RN: Cognitive trajectories after postoperative delirium. N Engl J Med 367: 30-39, 2012.

25. Li Z, Cao Y, Li L, Liang Y, Tian X, Mo N, Liu Y, Li M, Chui D and Guo X: Prophylactic angiotensin type 1 receptor antagonism confers neuroprotection in an aged rat model of postoperative cognitive dysfunction. Biochem Biophys Res Commun 449: 74-80, 2014.

26. Rundshagen I: Postoperative cognitive dysfunction. Dtsch Arztebl Int 111: 119-125, 2014.

27. Shoair OA, Grasso Ii MP, Lahaye LA, Daniel R, Biddle CJ and Slattum PW: Incidence and risk factors for postoperative cognitive dysfunction in older adults undergoing major noncardiac surgery: A prospective study. J Anaesthesiol Clin Pharmacol 31: 30-36, 2015.

28. Strøm C, Rasmussen LS and Steinmetz J: Practical management of anaesthesia in the elderly. Drugs Aging 33: 765-777, 2016.

29. Zhang L, Chen C, Wang L, Cheng G, Wu WW and Li YH: Awakening from anesthesia using propofol or sevoflurane with epidural block in radical surgery for senile gastric cancer. Int $\mathbf{J}$ Clin Exp Med 8: 19412-19417, 2015.

30. Akarsu Ayazoğlu T, Özensoy A, Geyik FD, Çalım M, Duman U and Candan MA: Effects of combined epidural analgesia with total intravenous anesthesia on risky patients underwent major abdominal surgery. Agri 27: 171-180, 2015.

31. Nishikawa K, Nakayama M, Omote K and Namiki A: Recovery characteristics and post-operative delirium after long-duration laparoscope-assisted surgery in elderly patients: Propofol-based vs. sevoflurane-based anesthesia. Acta Anaesthesiol Scand 48: $162-168,2004$.

32. Murata F, Iwade M, Hidano G, Tsunoda C, Nagata O and Ozaki M: Recovery from propofol anesthesia is delayed in hepatectomy patients due to altered pharmacodynamics. Masui 55: 150-157, 2006 (In Japanese).

33. Arevalo-Rodriguez I, Smailagic N, Roqué I Figuls M, Ciapponi A, Sanchez-Perez E, Giannakou A, Pedraza OL, Bonfill Cosp X and Cullum S: Mini-Mental State Examination (MMSE) for the detection of Alzheimer's disease and other dementias in people with mild cognitive impairment (MCI). Cochrane Database Syst Rev 3: CD010783, 2015.

34. Fu M and Li D: General anesthesia combined with epidural anesthesia on the postoperative cognitive functions in pregnant women with dystocia. Exp Ther Med 16: 1149-1152, 2018.

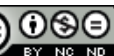

This work is licensed under a Creative Commons Attribution-NonCommercial-NoDerivatives 4.0 International (CC BY-NC-ND 4.0) License. 